\title{
How Do I Manage Penetrating Neck Trauma?
}

\author{
Hyun Min Cho \\ Department of Trauma and Surgical Critical Care, Pusan National University Hospital, Busan, Korea
}

Penetrating neck trauma (PNT) refers to neck injuries induced by gunshot wounds, stab wounds, or impaling objects, such as glass or sharp debris. Before World War II, all PNTs were treated conservatively and mortality rate was as high as $35 \%$. A mandatory surgical approach was adopted in an attempt to reduce the high death rates associated with PNTs and remained widely accepted well into the 1990s. This approach resulted in reduced mortality rates. Although the low mortality rate became obvious, the rate of negative surgical explorations was unacceptably high. Therefore the concept of selective surgical management which offers a spectrum of diagnostic approaches came from this observation. PNT, defined by platysma violation, comprises a sizable number of traumatic injuries in adults and remains a difficult management issue. While surgical intervention is necessary in approximately $15 \%-20 \%$ of PNT, the management of stable patients remains a source of debate. Although a variety of options are available for the management of PNT, physicians should try to find the best way to treat these injuries.

Key Words: Wounds, penetrating; Neck injuries

(Trauma Image Proced 2017(1):1-6)

\section{INTRODUCTION}

Penetrating neck trauma (PNT) involves a missile or sharp object penetrating the skin and violating the platysma layer of the neck. This includes gunshot wounds, stab or puncture wounds, and impalement injuries (1-3). PNT represents approximately 5\%-10\% of all trauma cases that present to the emergency department $(4,5)$. Approximately $30 \%$ of these cases are accompanied by injury outside of the neck zones as well $(4,5)$. The current mortality rate in civilians with PNT is $3 \%-6 \%(4,5)$. During World War II, the mortality rate was $7 \%$, and in World War I, it was $11 \%(4,5)$. Higher mortality rates occur with injuries to large vessels, such as the carotid or subclavian arteries and veins. Recent experience in the treatment of casualties from the Iraq War at Walter Reed Army Medical Center reported the common carotid artery as the most frequently injured cervical vessel (6).

The neck is located between the head and torso, and contains vital structures, such as the trachea, esophagus, carotid artery, jugular veins, and spinal cord. While relatively uncommon in comparison with other mechanisms of injury, the potential morbidity of PNT is apparent because of the high density of vital structures confined to a relatively small and poorly protected area (7). Although PNT was estimated in $10 \%$ of all trauma patients, overall mortality rates were estimated at $3 \%$ $6 \%$, most commonly as a result of injury to vascular structures and hemorrhage (8-10).

Received: March 3, 2017 Revised: April 1, 2017 Accepted: April 1, 2017

Correspondence to: Hyun Min Cho, Department of Trauma and Surgical Critical Care, Pusan National University Hospital, Busan, Korea 179, Gudeok-ro, Seo-gu, Busan, Korea

Tel: 82-51-240-7369, Fax: 82-51-240-7719, E-mail: csking1@daum.net

Copyright (c) 2017 Korean Association for Research, Procedures and Education on Trauma. All rights reserved.

(c) This is an open-access article distributed under the terms of the Creative Commons Attribution Non-Commercial License (http://creativecommons.org/ licenses/by-nc/4.0) which permits unrestricted noncommercial use, distribution, and reproduction in any medium, provided the original work is properly cited 
Successful management of PNT depends on a clear understanding of the anatomy of the neck (11). Anatomically, the neck can be divided into three major zones according to Monson et al. (12). These three zones allow for easy initial assessment and management, including surgical exploration and hemorrhage control. Zone I extends from the clavicle to the cricoid cartilage, zone II from the cricoid cartilage to the mandibular angle, and zone III from the mandibular angle to the base of the skull. Particularly in zone II, many important structures (the carotid arteries, jugular veins, thyroid, larynx, trachea, and esophagus) are present and unprotected. Therefore, damage to zone II is very fatal and often requires immediate surgical intervention (13). However, the usefulness of the zone concept of PNT has declined in recent years $(9,14)$.

\section{MAIN SUBJECT}

PNTs are very critical because of the high concentration of the airway structures, arteries, and veins. The platysma is a thin muscular sheet that surrounds the superficial fascia of the neck, and its integrity determines whether a PNT is superficial or deep. The possibility for injury to a vital organ exists when this structure is penetrated. If the platysma is penetrated in the initial survey, active surgical intervention is required (15).

As previously stated, the neck can be divided into three zones according as the anatomic landmarks. Each zone has a group of vital structures that can be injured and may determine the type of trauma management. Zone I is the horizontal area between the clavicle/suprasternal notch and cricoid cartilage encompassing the thoracic outlet structures. The proximal common carotid, vertebral, and subclavian arteries, and the trachea, esophagus, thoracic duct, and thymus are located in zone I. Zone II is the area between the cricoid cartilage and the mandibular angle. It contains the internal and external carotid arteries, jugular veins, pharynx, larynx, esophagus, recurrent laryngeal nerve, spinal cord, trachea, thyroid, and parathyroids. Zone III is the area that lies between the mandibular angle and the base of the skull. It includes the distal extracranial carotid and vertebral arteries and the uppermost segments of the jugular veins.

\section{Presentation}

1. Significant injury to vital structures of the neck can be indicated by the following clinical manifestations:

- Dysphagia: Tracheal and/or esophageal injury

- Hoarseness: Tracheal and/or esophageal injury (especially recurrent laryngeal nerve)

- Oronasopharyngeal bleeding: Vascular, tracheal, or esophageal injury

- Neurologic deficit: Vascular and/or spinal cord injury

- Hypotension: Nonspecific; may be related to the neck injury or may indicate trauma elsewhere

2. Proposed hard signs of airway injury include the following:

- Subcutaneous emphysema: Tracheal, esophageal, or pulmonary injury

- Air bubbling through the wound

- Stridor or respiratory distress: Laryngeal and/or esophageal injury

3. Several so-called hard signs that strongly indicate vascular injury are as follows:

- Hematoma (expanding): Vascular injury

- Active external hemorrhage from the wound site: Arterial vascular injury

- Bruit/thrill: Arteriovenous fistula

- Pulselessness/pulse deficit

- Distal ischemia (neurologic deficit in this case)

Evaluation of a patient with PNT should always start with the guideline of the advanced trauma life support (ATLS), a paradigm that begins with a directed primary survey emphasizing airway, breathing, and circulation (“ABC"). After patients are stabilized, they undergo a secondary survey that includes a complete history and thorough physical examination. These steps, together 
with studies discussed during the workup, are used to identify the likely injury complex and to direct further treatment or diagnostic testing.

Computed tomographic angiography (CTA) is generally considered the initial diagnostic method to evaluate the injured organs in PNTs (9). Woo et al. (6) reports that the use of CTA is associated with fewer operative explorations, fewer negative explorations, and reduced use of invasive studies, such as conventional angiography. Physical examination findings supplemented by CTA should have a prominent role in the selective management of PNTs. CTA has replaced angiography as the initial study of choice in the vascular evaluation of neck injuries (16). Comprehensive physical examination with CTA is adequate for identifying and excluding vascular and aerodigestive injuries after PNTs (17). As the accuracy of CTA in the diagnosis of critical structural damage increases, the choice of the clinical decision, especially the decision of the surgical exploration is getting easier (7). At a trauma center with experienced staff, the frequency of negative exploration for PNTs can be minimized by the selective neck exploration $(16,17)$.

The standard management is immediate surgical exploration for patients who present with signs and symptoms of shock and continuous hemorrhage from the neck wound (9). Moreover, all patients with active bleeding, expanding hematoma, shock, massive subcutaneous emphysema, or significant airway compromise should be directly admitted to the operating room and are surgically explored, regardless of the zone of injury $(9,14)$. The following specific injuries must be confirmed and treated during neck exploration: vascular (carotid artery, vertebral artery, and jugular vein), laryngotracheal, esophageal, nerve, thoracic duct, and thyroid injuries. Currently, mandatory surgical exploration for all injuries that penetrate the platysma is no longer practiced (9). However, mandatory exploration of all neck wounds may be the best policy in an environment in which routine serial examinations are not possible (11).

The type of incision depends on the injured zone and structures at risk of injury. An additional consideration is proper exposure to gain adequate proximal and distal control of the involved blood vessels. The standard neck incision, parallel to the medial border of the sternocleidomastoid muscle, can be used for most zone II injuries and can be extended cephalad for zone III injuries, specifically for injuries to the distal carotid or vertebral arteries. The standard neck incision can be transversely extended to the opposite side for bilateral injuries. A transverse or collar-type incision can be performed for suspected injuries traversing the cervical region, providing exposure to both sides and obviating the need for bilateral neck incisions. A supraclavicular incision provides good exposure for zone I injuries. Removal of the head of the clavicle with an oscillating saw may provide better exposure. In conjunction, an anterolateral thoracotomy incision can be used for thoracic inlet injuries. The trapdoor or open-book thoracotomy includes a median sternotomy with an anterolateral extension and a supraclavicular extension for more exposure of zone I injuries. The specific injuries described below must be confirmed and treated during neck exploration.

\section{Surgical consideration}

1. Carotid artery injuries are the most common, with an incidence of approximately $9 \%(1,2)$. They also pose one of the most immediate life-threatening situations. The objective of surgical care is to stop the hemorrhage, maintain the cerebral blood flow, and preserve neurologic function. Arteriorrhaphy, vein patch, or segmental repair with an autologus reversed saphenous vein graft can be performed to repair the injury. Arterial repair has been shown to have lower morbidity and mortality rates than ligation $(6,10,16)$. The presence of neurologic deficits, coma, and shock, especially preoperatively, are poor prognostic signs but are not absolute contraindications for carotid artery repair. Carotid ligation has been advocated in patients who are comatose with no evidence of antegrade flow in the internal carotid artery. Ligation can also be an option when uncontrollable hemorrhage is present 
and temporary shunt placement is technically difficult.

2. Vertebral artery injuries have been diagnosed with increasing frequency with the liberal use of arteriography, particularly four-vessel angiography. The treatment of choice in the well-perfused patient is expectant management. Definitive intervention is indicated if a pseudoaneurysm, arteriovenous fistula, or persistent bleeding is documented. Surgical repair can be performed, but if the circle of Willis is patent, ligation is always an option. Angiographic embolization has advantages for this difficult-toaccess artery, but distal control remains a problem.

3. For the jugular vein injury, there are various surgical options as the condition of the injuries and patient as follows; simple lateral closure, resection and reanastomosis, or saphenous vein interposition graft. Repairing at least one-side can be mandatory if both internal jugular veins are injured. Contrarily, the external jugular vein can be ligated without any adverse effects.

4. Laryngotracheal injuries are also common, with a combined incidence of $10 \%$ among PNT $(1,4,14)$. Tracheal injuries can be primarily repaired using one layer of sutures. Adjacent omohyoid or sternocleidomastoid muscles should be interposed when esophageal and arterial repair are concomitantly performed to prevent fistula formation. A tracheostomy is indicated when injury is severe, but performing it via the site of surgical repair should be avoided. A soft intralaryngeal stent in cases of extensive disruption of the cartilaginous support is recommended.

5. Esophageal injuries are the third most common in PNTs $(6 \%)(1,4,14)$. Signs and symptoms of dysphagia, hematemesis, subcutaneous crepitus, retropharyngeal air, and injuries to adjacent structures are strong indicators of esophageal injury. Early diagnosis lessens the probability of delayed treatment and missed injury, which can be devastating (e.g., mediastinitis). The recommended management of esophageal injury is primary repair and adequate drainage. Oral feeding may be initiated after a barium swallow study shows no evidence of a leak. For extensive injuries or in cases of delayed diagnosis with significant infection, the better option is to establish a controlled fistula with catheter drainage or an esophagostomy. Hypopharyngeal wounds can sometimes be treated with a nasogastric tube for feeding and parenteral antibiotics. Parenteral nutrition via feeding jejunostomy can be performed.

6. Nerve injuries account for approximately $1 \%-3 \%$ of PNT $(1,4,14)$. Injury to the vagus, recurrent laryngeal nerve, or brachial plexus should be primarily repaired when it is feasible (e.g., in a well-perfused patient without active hemorrhage). Spinal cord injury caused by PNT can be expectantly managed. Steroids have not been shown to be beneficial in PNTs.

7. Thoracic duct injuries, albeit difficult to demonstrate, can occur. They should be ligated to prevent chylous fistula and infections in the neck and mediastinum.

8. Thyroid injuries are uncommon despite the size and location of the thyroid in the neck. Injuries can cause significant bleeding that is often controlled with direct pressure or suture ligation. Extensive injury may require an ipsilateral lobectomy to resolve bleeding.

9. Severe parotid injury is rare but a parotidectomy can be performed when the injury accompanies by vessel injuries.

PNTs may be extremely fatal because of the high concentration of vital structures in the neck, despite a low abbreviated injury scale (AIS) score. Therefore, early " $\mathrm{ABC}$ " is very important in PNT. The initial evaluation of a trauma patient begins with "ABC" of trauma management: establish a secure airway, breathing/r espiration, and volume resuscitation. Also, particular importance should be placed on the airway because bleeding within the tight compartmentalized spaces of the neck may appear externally quiescent, but may cause progressive airway compromise and eventual complete 
obstruction (14). There is evidence to suggest that the hard signs of airway injury are more reliable and result in fewer negative operative explorations compared than the hard signs of vascular injury. The rate of negative exploration for patients with hard signs of vascular injury varies widely, but it may be estimated at $10 \%$ $(1,10,14)$. However, series that report these cases as "nonsignificant" injury or as negative explorations lack clear definition, and it is difficult to draw any useful conclusion from the data.

\section{CONCLUSION}

PNT is an important area of trauma care that has undergone evolution in the recent past. A remarkable number of changes have occurred in the treatment paradigm as new technologies have developed and surgeons have explored the outcomes from different treatment protocols. Therapy has evolved from no treatment (before effective anesthesia and instrumentation), to nonoperative management, to routine exploration, to selective exploration, and adjunctive invasive or noninvasive assessment. PNTs remain challenging, as a number of important structures exist in a small area, and injury to any of these structures may not be readily apparent. The assessment and management of PNTs remain controversial. However, not only meticulous clinical examination but also CTA are essential for evaluating PNT patients. The standard management is immediate surgical exploration for patients who present with active bleeding, expanding hematoma, shock, massive subcutaneous emphysema, or significant airway compromise, regardless of the zone of injury $(9,14)$. It is most important for a PNT patient to keep in mind the basics of treating trauma, early " $\mathrm{ABC}$ " of trauma management.

\section{Conflict of Interest Statement}

No potential conflict of interest relevant to this article was reported.

\section{REFERENCES}

1. Brennan J, Lopez M, Gibbons MD, Hayes D, Faulkner J, Dorlac WC, et al. Penetrating neck trauma in Operation Iraqi Freedom. Otolaryngology--head and neck surgery : official journal of American Academy of OtolaryngologyHead and Neck Surgery. 2011;144(2):180-5.

2. Dubois-Marshall S, De Kock S. Two days with a broken knife blade in the neck--an interesting case of Horner's syndrome. Emergency medicine journal : EMJ. 2011;28(7): 629-31.

3. Gupta B, Gulati A, Gupta D. A rare presentation of pellet injury in the neck. ISRN surgery. 2011;2011:306126.

4. McConnell DB, Trunkey DD. Management of penetrating trauma to the neck. Advances in surgery. 1994;27:97-127.

5. Thal ER, Meyer DM. Penetrating neck trauma. Current problems in surgery. 1992;29(1):1-56.

6. Woo K, Magner DP, Wilson MT, Margulies DR. CT angiography in penetrating neck trauma reduces the need for operative neck exploration. The American surgeon. 2005;71(9):754-8.

7. Kasbekar AV, Combellack EJ, Derbyshire SG, Swift AC. Penetrating neck trauma and the need for surgical exploration: six-year experience within a regional trauma centre. The Journal of laryngology and otology. 2017; 131(1):8-12.

8. Brennan J. Head and neck trauma in Iraq and Afghanistan: different war, different surgery, lessons learned. The Laryngoscope. 2013;123(10):2411-7.

9. Burgess CA, Dale OT, Almeyda R, Corbridge RJ. An evidence based review of the assessment and management of penetrating neck trauma. Clinical otolaryngology : official journal of ENT-UK ; official journal of Netherlands Society for Oto-Rhino-Laryngology \& Cervico- Facial Surgery. 2012;37(1):44-52.

10. Mahmoodie M, Sanei B, Moazeni-Bistgani M, Namgar M. Penetrating neck trauma: review of 192 cases. Archives of trauma research. 2012;1(1):14-8.

11. Thompson EC, Porter JM, Fernandez LG. Penetrating neck trauma: an overview of management. Journal of oral and maxillofacial surgery : official journal of the American Association of Oral and Maxillofacial Surgeons. 2002; 60(8):918-23.

12. Monson DO, Saletta JD, Freeark RJ. Carotid vertebral trauma. The Journal of trauma. 1969;9(12):987-99.

13. Koehler SA, Shakir A, Williams KE. Accidental through and through penetrating injury to the neck. The American journal of forensic medicine and pathology. 2011;32(1): 17-9. 
14. Bell RB, Osborn T, Dierks EJ, Potter BE, Long WB. Management of penetrating neck injuries: a new paradigm for civilian trauma. Journal of oral and maxillofacial surgery : official journal of the American Association of Oral and Maxillofacial Surgeons. 2007;65(4):691-705.

15. Miller RH, Duplechain JK. Penetrating wounds of the neck. Otolaryngologic clinics of North America. 1991; 24(1):15-29.
16. Schroeder JW, Baskaran V, Aygun N. Imaging of traumatic arterial injuries in the neck with an emphasis on CTA. Emergency radiology. 2010;17(2):109-22.

17. Shiroff AM, Gale SC, Martin ND, Marchalik D, Petrov D, Ahmed HM, et al. Penetrating neck trauma: a review of management strategies and discussion of the 'No Zone' approach. The American surgeon. 2013;79(1):23-9. 